\title{
A Possible Second Site for Hydroxyapatite Dissolution in Acidic Media ${ }^{1}$
}

\author{
MAHDI B. FAWZI, JEFFREY L. FOX, ${ }^{2}$ MAHENDRA G. DEDHIYA, \\ WILLIAM I. HIGUCHI, AND JOHN J. HEFFERREN \\ College of Pharmacy, The University of Michigan, Ann Arbor, Michigan 48109
}

Received October 7, 1977; accepted May 30, 1978

\begin{abstract}
Dissolution of human dental enamel and of synthetic hydroxyapatite pellets in completely unsaturated acetate buffer solutions was previously shown to follow a model in which a single dissolution site was employed. The present investigation reports similar studies carried out under the clinically more relevant conditions of dissolution into solutions partially saturated with respect to hydroxyapatite, and it is shown that under these conditions the dissolution kinetics may be described by a site which has properties different from the site responsible for dissolution into completely unsaturated solutions. The properties of this second site were consistent with experiments in which bulk solution conditions (degree of partial saturation, $\mathrm{Ca} / \mathrm{P}$ ratio) and effective diffusion layer thickness were varied. Thus, dissolution kinetics studies indicate that hydroxyapatite crystals have at least two different types of sites that are important in dissolution.
\end{abstract}

\section{INTRODUCTION}

In previous investigations we have studied the dissolution rate behavior of human dental enamel and synthetic hydroxyapatite in weak acid buffers. These investigations included studies of the influences of $\mathrm{pH}$, common ion, fluoride, and trace elements on the dissolution rate behavior $(3,4,6)$.

Most of the previous studies were conducted under sink conditions; that is, saturation of the acidic media with respect to hydroxyapatite was not permitted to exceed about $1 \%$. Under these conditions it was found (9) that the dissolution rate behavior of hydroxyapatite $\left(\mathrm{NBS}^{3}\right)$ could be described quantitatively and mechanistically by two parameters. The first of them is an

\footnotetext{
1 This investigation was supported by NIDR grant DE-01830.

${ }^{2}$ To whom correspondence should be addressed.

${ }^{3}$ NBS hydroxyapatite is synthesized by the slow addition of pure phosphoric acid solution into a boiling $\mathrm{CO}_{2}$-free, $\mathrm{CaO}$ solution. The resulting precipitate is then digested for 2 days in the boiling reaction solution. The residue is washed, filtered, and then dried at $105^{\circ} \mathrm{C}$.
}

activity product function:

$$
K_{\mathrm{HAP}}=\left(a_{\mathrm{Ca}^{++}}\right)^{10}\left(a_{\mathrm{PO}_{4}} \equiv\right)^{6}\left(a_{\mathrm{OH}^{-}}\right)^{2}
$$

of $10^{-125 \pm 1}$, which expresses the apparent solubility driving source for the dissolution under sink conditions and the second parameter is a first order rate constant $k$ which is the measure of the rate of ion disengagement at the crystal surface. Essentially all of the experiments conducted under sink conditions have been quantitatively explainable by a physical model consisting of this dissolution site, along with the appropriate considerations of multicomponent diffusion and rapid chemical equilibrium in the diffusion layer.

Although these studies were a major step in the understanding of enamel and synthetic HAP (NBS) dissolution under sink conditions, it was apparent that they could not provide an explanation for the more complex process of in vivo caries formation. This process occurs in an oral environment partially saturated with respect to hydroxyapatite and is characterized by an initial "white spot" formation involving subsurface demineralization throughout a 
"zone" of dissolution rather than simple surface dissolution characterized by recession of the surface.

It is the purpose of the present communication to describe the results of the investigations of the dissolution rate behavior of the (NBS) hydroxyapatite under moderate partial saturation conditions in acidic buffers using the rotating disk method and following the physical model recently developed by Wu et al. (9) for data analysis. As will be seen, under partial saturation conditions, corresponding to a bulk solution activity product of $K_{\mathrm{HAP}}=\left(a_{\mathrm{Ca}^{2+}}\right)^{10}\left(a_{\mathrm{PO}_{4}}\right)^{6}$ $\times\left(a_{\mathrm{OH}^{-}}\right)^{2}=10^{-137}$ to $10^{-127}$, the dissolution rate is largely governed by a second new site different from that which dominates the dissolution rate under sink conditions.

\section{Physical Model for Dissolution of HAP Pellets}

We employed the physical model developed by Wu et al. (9) for hydroxyapatite dissolution under sink conditions. In this model an enamel block or a compressed hydroxyapatite pellet is treated as a matrix of hydroxyapatite crystals separated by intercrystalline spaces that are permeated with water. The matrix is separated from the bulk acid buffer solution by a diffusion layer, and the effective diffusion layer thickness $h$ is calculated from hydrodynamic considerations using a treatment developed by Levich (5) and corrected for the rotating disk experimental system (9). Essentially the model consists of solving the problem of simultaneous diffusion and crystal dissolution in the matrix and aqueous diffusion layer. The treatment used here is similar to that used by $\mathrm{Wu}$, the only difference being the fact that the bulk solutions used here were partially saturated.

\section{EXPERIMENTAL PROCEDURE}

\section{Hydroxyapatite Synthesis}

Radiolabeled and nonradiolabeled synthetic hydroxyapatite samples were pre- pared using the procedures developed by Moreno et al. (7). A phosphoric acid solution is slowly added to a boiling $\mathrm{CO}_{2}$ free $\mathrm{CaO}$ suspension. The resulting precipitate is then digested for 2 days in the boiling reaction solution. The residue is then washed with boiling double-distilled water for a few hours, filtered, rinsed with acetone, and then dried at $105^{\circ} \mathrm{C}$ for $24 \mathrm{hr}$.

\section{Hydroxyapatite Pellet Preparation}

About $40 \mathrm{mg}$ of hydroxyapatite preequilibrated in a humidity chamber containing saturated potassium nitrate aqueous solution was directly compressed in a $0.25^{\prime \prime}$ diameter die with a force of $10,000 \mathrm{lb}$ using a laboratory press. The pellet was then ejected from the die and mounted on a pellet holder with melted paraffin so that only one flat surface of the apatite pellet was exposed.

\section{Preparation of Buffers}

The acid buffer solutions were prepared by mixing calculated quantities of the acid and their salts. Partially saturated buffer solutions were made by adding predetermined amounts of calcium chloride or sodium dihydrogen phosphate. In both cases, calculated amounts of sodium chloride were added to maintain constant ionic strength of $0.5 \mu \mathrm{m}$. The $\mathrm{p} K_{\mathrm{HAP}}$ of these solutions were calculated by a computer program developed by Fox (1).

\section{Analytical Chemistry Procedures}

Methods for the analysis of phosphate and calcium used in the present work have already been described (6). Calcium was determined by atomic absorption spectrophotometry and phosphate by a colorimetric procedure. ${ }^{45} \mathrm{Ca}$ was analyzed by a scintillation counter (Beckman liquid scintillation system. Beckman Instruments, Inc., Fullerton, California). 


\section{Experimental Procedure}

Rotating disk apparatus. Figure 1 is a schematic representation of the experimental rotating disk dissolution apparatus. The pellet was fixed in the center of the disk by melted paraffin. Prior to an experiment this pellet was soaked in double-distilled water overnight. This disk assembly was connected to a constant speed rotating mechanism. The motor was firmly suspended by means of clamps from a series of bars which were rigidly fastened to the laboratory bench. The ratio of the flask diameter to disk diameter was 2.5 which exceeded the minimum 2.4 ratio recommended by Riddiford ( 8 ). The distance from the disk to the walls and bottom of the flask exceeded $0.5 \mathrm{~cm}$, and the disk was immersed to a level which exceeded $0.5 \mathrm{~cm}(8)$.

The dissolution rate experiments were run as follows. An acid buffer solution or the partially saturated buffer $(50 \mathrm{ml})$ was accurately measured and placed into the jacketed $100-\mathrm{ml}$ beaker and allowed to attain thermal equilibrium at $30^{\circ} \mathrm{C}$. Then the flask containing the solution was raised, using a lab jack, until the pellet surface was immersed. At the moment of contact, the motor and a timer were simultaneously started. Then the pellet holder was lowered to its final position. An aliquot of sample (about $1 \mathrm{ml}$ ) was withdrawn by pipet at suitable intervals. The solution was replaced by an equal volume of the same buffer solution maintaining a constant volume. The samples were diluted and analyzed for calcium and/or phosphate.

\section{General Considerations of the Experimental System}

In these studies three different samples were employed. The first was an authentic (NBS) hydroxyapatite sample (received from the National Bureau of Standards). This was used to standardize the other two samples synthesized in our laboratory

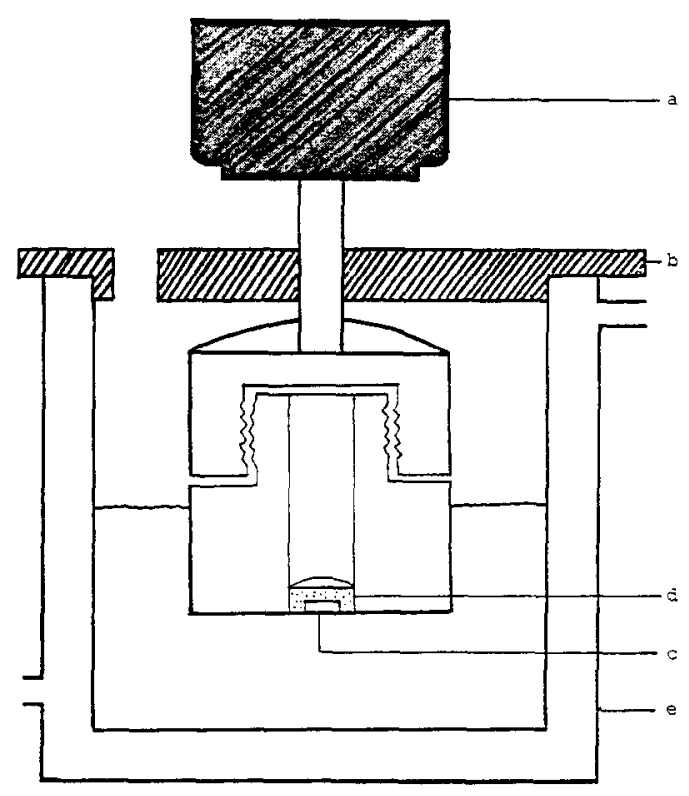

FIG. 1. Rotating disk experimental set up: a, constant speed motor; b, cover; c, hydroxyapatite pellet; $d$, paraffin; e, jacketed glass beaker.

following the procedure described by Moreno et al. (7).

The samples prepared in our laboratories were ${ }^{45} \mathrm{Ca}$-radiolabeled (NBS) hydroxyapatite and cold (NBS) hydroxyapatite. These samples were compared with the authentic (NBS) hydroxyapatite using infrared, X-ray, and elemental analysis to ensure that the experimental results would not be dependent on the source of hydroxyapatite used. In addition to these tests, the three samples were compared under our experimental conditions on a regular basis and all these pairs of comparable experiments showed an excellent agreement between our sample and the authentic (NBS) hydroxyapatite sample.

In most of the studies reported in this communication, the radioactive sample was used to insure high accuracy in measuring the rate of dissolution under partial saturation conditions. The error for the analysis in these experiments was consistently found to be less than $\pm 1 \%$ of the amount being measured. 


\section{Experimental Results}

The data obtained from dissolution experiments were plotted as amount of hydroxyapatite dissolved $(\mathrm{mg})$ in $50 \mathrm{ml}$ of buffer vs time. The amounts of hydroxyapatite dissolved were calculated on the basis of both calcium and phosphate analyses whenever both were possible. Good stoichiometry, i.e., congruent dissolution, was found in all the experiments. However, because of the somewhat better precision of the radiolabeled ${ }^{45} \mathrm{Ca}$ analysis, the data obtained by this method were used to calculate dissolution rates whenever possible.

A typical set of data showing the effect of changing the rotation speed on the dissolution rate is shown in Fig. 2. As can be seen from this data, there is only a very weak dependence of dissolution rate on rotation speed. The weakness of this de- pendence indicates that the dissolution rate is not limited purely by the diffusion process, but that surface solution kinetics also plays a rate-limiting role, more so than in the sink conditions of Wu et al. (9) where this kinetic limitation was of lesser importance.

Also, each of these pellets was examined visually and mechanically after its dissolution run and was found to have the softened texture indicative of zonal dissolution rather than simple surface dissolution. This zone softening was observed with all three samples of hydroxyapatite used in these studies and was more pronounced under conditions of higher partial saturation, although no quantitation of the softening was attempted in any of the experiments.

Because of the long-time periods required for each experiment and the zonal softening produced during the course of a

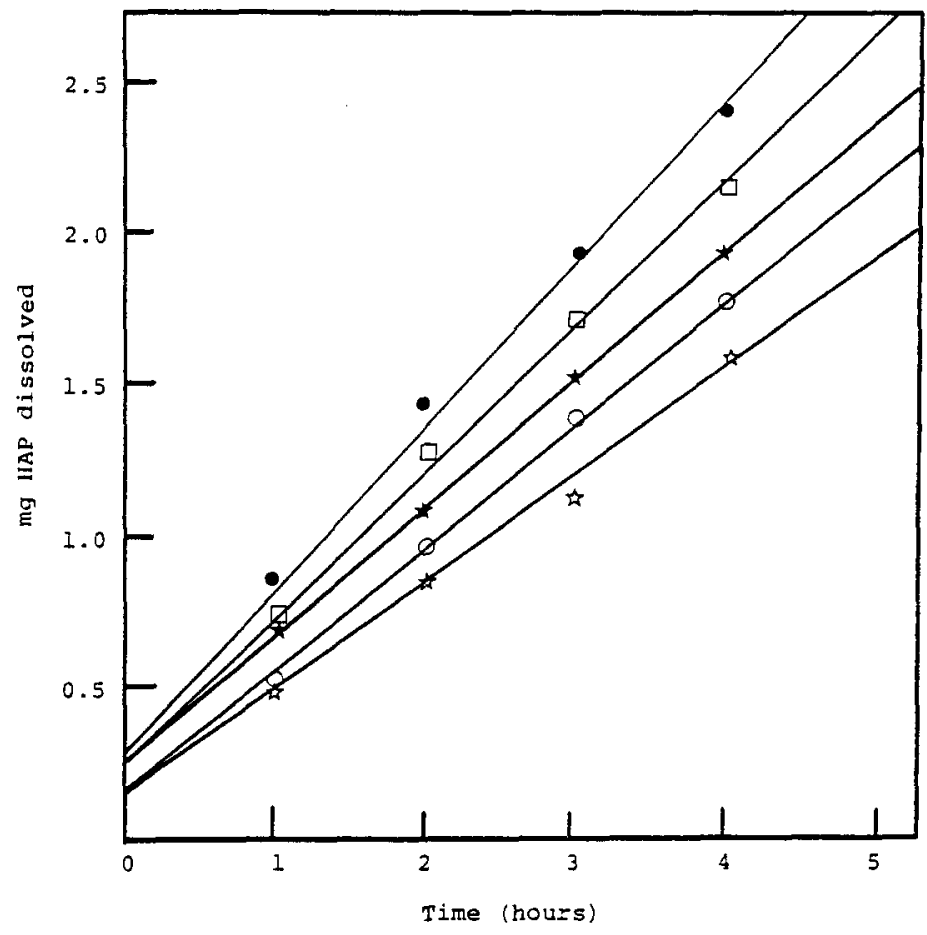

Fig. 2. Effect of rotation speed on the dissolution rates of ${ }^{45} \mathrm{Ca}$-HAP pellets $\left(A=0.317 \mathrm{~cm}^{2}\right)$. Bulk solution conditions: $0.1 \mathrm{M}$ acetate buffer at $\mathrm{pH} 4.5$ and $0.5 \mathrm{M}$ ionic strength, TCa $=\mathrm{TP}$ $=3.524 \times 10^{-3} M$ (16\% saturation). $\bullet, 900 \mathrm{rpm} ; \square, 500 \mathrm{rpm} ; \star, 250 \mathrm{rpm} ; O, 100 \mathrm{rpm} ;$ 放, $50 \mathrm{rpm}$. 
TABLE I

The Values of the Parameters Obtained under Sink and Partial Saturation Conditions

\begin{tabular}{llc}
\hline \multicolumn{1}{c}{ Bulk solution } & \multicolumn{1}{c}{$K_{\mathrm{HAP}^{a}}$} & \multicolumn{1}{c}{$k D^{\prime b}$} \\
\hline Sink conditions & \\
Partial saturation conditions & $10^{-125}$ & $44 \times 10^{-6}$ \\
\hline
\end{tabular}

${ }^{a} K_{\mathrm{HAP}}=\left(a_{\mathrm{Ca}^{2+}}\right)_{\mathrm{s}}^{10}\left(a_{\mathrm{PO}_{4} \mathrm{~s}_{\mathrm{s}}}\right)^{6}\left(a_{\mathrm{OH}^{-}}\right)_{\mathrm{s}}^{2}$.

${ }^{b} k=$ apparent first order rate constant; $D^{\prime}=$ diffusivity in the enamel or HAP pellet.

${ }^{c}$ Wu et al. (9).

single experiment, a new hydroxyapatite pellet was used for each dissolution run. It was found however, that repeat runs on the same pellet were always within experiment error of the first run. The pelletto-pellet variations were also small, as dissolution rates with different pellets under the same experimental conditions were almost always within $4 \%$ of each other.

The dissolution rate data were treated in a manner similar to that described by Wu et al. (9), and the values obtained for the dissolution site parameters were considerably different than those he obtained under sink conditions using identical experimental techniques. Since the uncertainty

\section{TABLE II}

The Relationship between the Bulk Solution Activity Product and the Actual Total Calcium and Phosphate in Solution

\begin{tabular}{ccccc}
\hline $\begin{array}{c}\text { Bulk solution } \\
\text { activity } \\
\text { product as } \\
\mathrm{p}_{\text {HAP }}{ }^{\mathrm{a}}\end{array}$ & $\begin{array}{c}\text { Solution } \\
\text { Ca to TP } \\
\text { ratio }\end{array}$ & \begin{tabular}{c} 
Concentration \\
\cline { 3 - 5 }$\times 10^{3} M$
\end{tabular} & $\begin{array}{c}\mathrm{TP} \\
\times 10^{3} M\end{array}$ & $\begin{array}{c}\text { Nominal } \\
\text { percentage } \\
\text { saturation }^{\circ} \\
(\%)\end{array}$ \\
\hline 138.2 & 1 & 0.852 & 0.852 & 4 \\
135.8 & 1 & 1.3 & 1.3 & 6 \\
132.9 & 1 & 1.704 & 1.704 & 8 \\
128.4 & 1 & 3.524 & 3.524 & 16 \\
128.4 & 10 & 8.356 & 0.8356 & 16 \\
128.4 & 0.1 & 1.48 & 14.80 & 16 \\
128.4 & 100 & 19.8 & 0.198 & 16 \\
\hline
\end{tabular}

${ }^{a}$ All these solutions are acetate buffer of $0.1 \mathrm{M}$ acetate, $\mathrm{pH}=4.5$, and their ionic strength is adjusted to $0.5 M$ by adding $\mathrm{NaCl}$.

${ }^{b} 100 \%$ saturation here is the thermodynamic $K_{\mathrm{sp}}$ determined by Moreno et al. (7). of the values of these parameters was much smaller than the difference between these parameters and those obtained by $\mathrm{Wu}$, it appeared that there was a new, second dissolution site on hydroxyapatite crystals with values of $K_{\mathrm{HAP}}$ and $k D^{\prime}$ as shown in Table I. Because this idea of a second dissolution site marked such a radical departure from previous thinking, a series of experiments were designed to critically test this idea.

Dissolution experiments were performed under varying degrees of partial saturation and varying $\mathrm{Ca} / \mathrm{PO}_{4}$ ratios. The degree of partial saturation is denoted by the

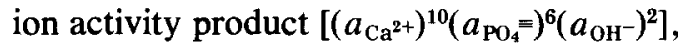
of the bulk solution. For a bulk solution with a $\mathrm{Ca} / \mathrm{PO}_{4}$ ratio of unity, ion products of $10^{-128.2}, 10^{-135.8}, 10^{-132.9}$, and $10^{-128.4}$ correspond to solutions that are approximately $4,6,8$, and $16 \%$ saturated, respectively, with respect to the equilibrium solubility of hydroxyapatite (see Tables II and

TABLE III

Selected Parameter Values used in the Calculations ${ }^{a}$

\begin{tabular}{|c|c|c|c|}
\hline Species & $\begin{array}{c}\text { Ionic } \\
\text { activity } \\
\text { coefficient } \\
(\gamma)\end{array}$ & Species & $\begin{array}{c}\text { Ionic } \\
\text { activity } \\
\text { coefficient } \\
(\gamma)\end{array}$ \\
\hline $\mathrm{H}_{3} \mathrm{PO}_{4}$ & 1.0 & $\mathrm{Ca}^{2+}$ & 0.33 \\
\hline $\mathrm{H}_{2} \mathrm{PO}_{4}$ & 0.44 & $\mathrm{CaAc}^{+}$ & 0.80 \\
\hline $\mathrm{HPO}_{4}=$ & 0.15 & $\mathrm{CaHPO}_{4}$ & 1.0 \\
\hline $\mathrm{PO}_{4} \equiv$ & 0.029 & $\mathrm{CaH}_{2} \mathrm{PO}_{4}^{+}$ & 0.44 \\
\hline HAC & 1.0 & $\mathrm{H}^{+}$ & 0.8 \\
\hline \multirow[t]{3}{*}{$\mathrm{Ac}^{-}$} & 0.80 & $\mathrm{OH}^{-}$ & 0.69 \\
\hline & & \multicolumn{2}{|c|}{ Equilibrium constant } \\
\hline & Reaction & $\begin{array}{c}K_{\mathrm{a}} \\
\text { (thermo- } \\
\text { dynamic) }\end{array}$ & $\begin{array}{c}\mathrm{p} K_{\mathrm{a}} \\
\text { (corrected } \\
\text { for } \gamma \mathrm{s} \text { ) }\end{array}$ \\
\hline $\mathrm{H}_{3} \mathrm{PO}_{4}$ & $\rightleftharpoons \mathrm{H}_{2} \mathrm{PO}_{4}^{-}+\mathrm{H}^{+}$ & $6.98 \times 10^{-3}$ & 1.70 \\
\hline $\mathrm{H}_{2} \mathrm{PO}_{4}^{-}$ & $\rightleftharpoons \mathrm{HPO}_{4}=+\mathrm{H}^{+}$ & $6.40 \times 10^{-8}$ & 6.63 \\
\hline $\mathrm{HPO}_{4}=$ & $\rightleftharpoons \mathrm{PO}_{4}+\mathrm{H}^{+}$ & $4.73 \times 10^{-13}$ & 11.51 \\
\hline HAc & $\rightleftharpoons \mathrm{H}^{+}+\mathrm{Ac}^{-}$ & $1.75 \times 10^{-5}$ & 4.56 \\
\hline $\mathrm{CaAc}^{+}$ & $\rightleftharpoons \mathrm{Ca}^{2+}+\mathrm{Ac}^{-}$ & 0.295 & 0.0485 \\
\hline $\mathrm{CaHPO}_{4}$ & $\rightleftharpoons \mathrm{Ca}^{2+}+\mathrm{HPO}_{4}=$ & $3.92 \times 10^{-3}$ & 1.10 \\
\hline $\mathrm{CaH}_{2} \mathrm{PO}_{4}^{+}$ & $\rightleftharpoons \mathrm{Ca}^{2+}+\mathrm{H}_{2} \mathrm{PO}_{4}^{-}$ & $1.98 \times 10^{-1}$ & 0.220 \\
\hline $\mathrm{HOH}$ & $\rightleftharpoons \mathrm{H}+\mathrm{OH}$ & $1.8 \times 10^{-16}$ & 15.49 \\
\hline
\end{tabular}

\footnotetext{
${ }^{a}$ Ionic strengths adjusted to 0.50 with $\mathrm{NaCl}$.
} 


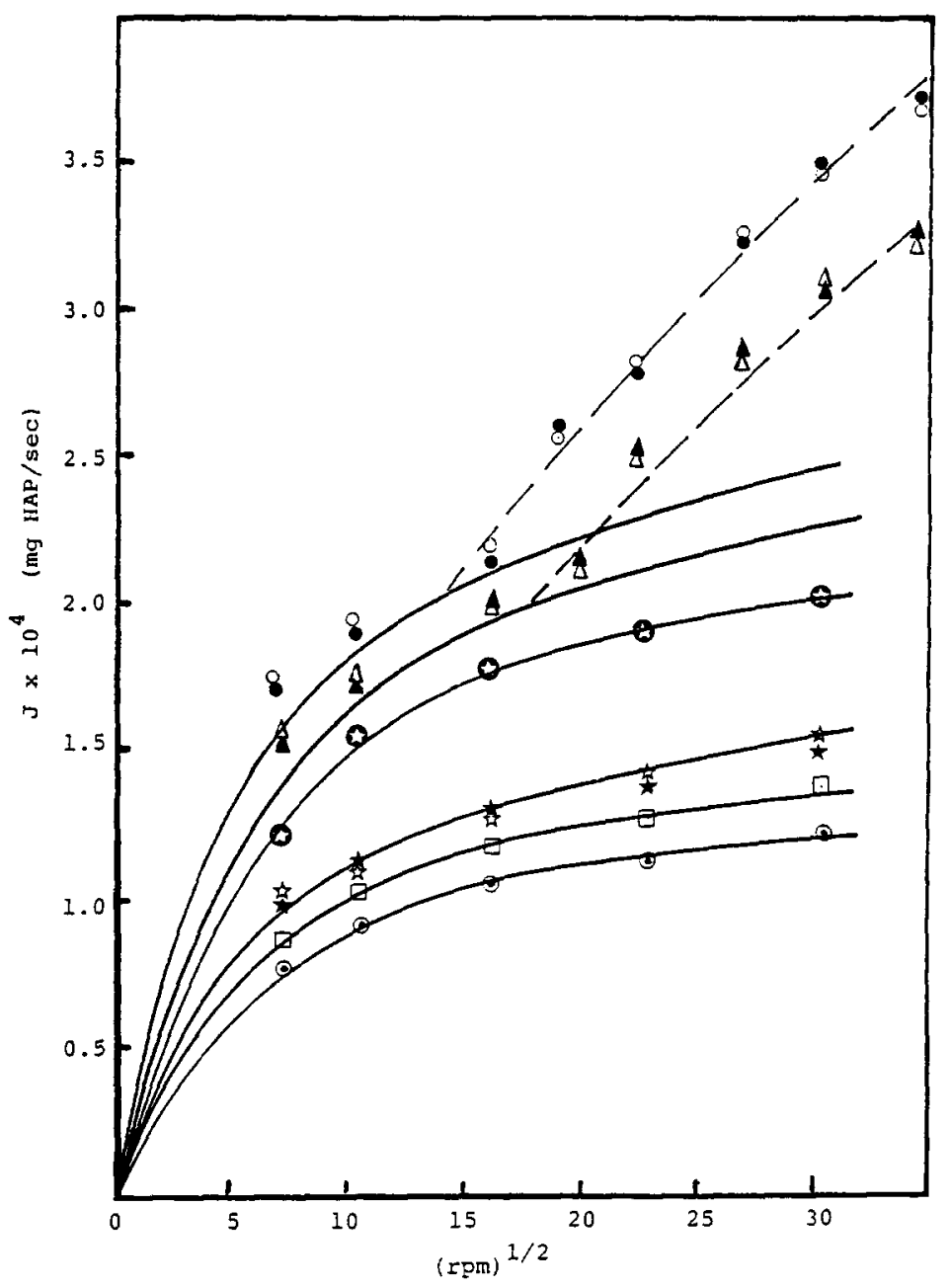

FIG. 3. Comparison between the observed and the predicted effect of stirring speed on the dissolution rate, $J$, of ${ }^{45} \mathrm{Ca}-\mathrm{HAP}$ pellets $\left(A=0.317 \mathrm{~cm}^{2}\right)$. Bulk solution conditions: $0.1 M$ acetate buffer at $\mathrm{pH} 4.5$ and $0.5 \mathrm{M}$ ionic strength. (-) partial saturation site prediction; (-- ) deviation from partial saturation prediction.

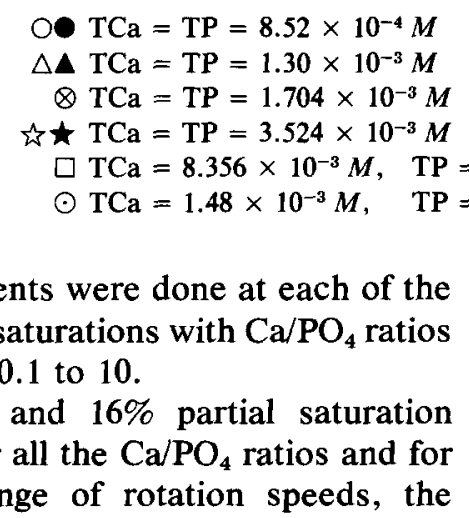

\begin{tabular}{r} 
\% saturation \\
\hline 4 \\
6 \\
8 \\
16 \\
16 \\
16
\end{tabular}

III). Experiments were done at each of the above partial saturations with $\mathrm{Ca} / \mathrm{PO}_{4}$ ratios varying from 0.1 to 10 .

For the 8 and $16 \%$ partial saturation conditions for all the $\mathrm{Ca} / \mathrm{PO}_{4}$ ratios and for the entire range of rotation speeds, the experimental results were consistent with the single $K_{\mathrm{HAP}}$ and $k D^{\prime}$ pair (see Fig. 3). This represented some 30 different sets of experimental conditions. The fact that experiment and theory were consistent (i.e., agreed well) does not necessarily indicate 
that the parameters used in the model are accurately known, however. The two parameters are, in fact, quite dependent on each other so that there is some uncertainty in their values.

For the 4 and $6 \%$ partial saturation the dissolution rates at the lower rotation speeds employed $(<200 \mathrm{rpm})$ were consistent with these same parameters. At higher rotation speeds, however, the dissolution into the 4 and $6 \%$ solutions was consistent with the parameters of the sink site described by Wu et al. (9). This break in the dissolution rate vs rotation curve for 4 and $6 \%$ partial saturation is indicative of a transition from one-site only dissolution kinetics to two-site kinetics and is fully consistent with the postulated properties of the dissolution sites. A detailed discussion of the two-site model appears in a separate paper by Fox et al. (2).

\section{DISCUSSION}

The experimental data indicate that under the higher partial saturation conditions employed in this study the rate of dissolution is governed by a site distinctly different from that found by $\mathrm{Wu}$ and co-workers (9) to govern dissolution under sink conditions. In determining the $\mathrm{p} K_{\mathrm{HAP}}$ for this site, it was found that the value that gave the best fit when considering all the data was about 121.8. It was found that values in this range were always obtained independently of what reasonable values were chosen for the effective diffusivity and the activity coefficients. However, if only the $16 \%$ data were used in obtaining these parameters, an equally good fit is given by a $\mathrm{p} K_{\mathrm{HAP}}$ value of 120 . The inclusion of the $8 \%$ data makes the fit sharper with a value of about 121.8. Extensive manipulation of these parameters (2) shows that although exact values for the $\mathrm{p} K_{\mathrm{HAP}}$ for the two sites are elusive at this point, the end result of any fitting procedure is the conclusion that the $\mathrm{p} K_{\mathrm{HAPS}}$ of the sites differ by several units.
Dissolution experiments in both 16 and $0 \%$ saturated solutions have shown that block enamel and the NBS hydroxyapatite used in this study exhibit essentially the same dissolution behavior (Fawzi et al., unpublished). It is therefore probable that the two sites responsible for dissolution of the synthetic hydroxyapatite are also responsible for the dissolution of human dental enamel. In particular, the single site responsible for dissolution of hydroxyapatite under partial saturation conditions is very likely the site that is important in the clinical development of carious lesions. The properties of this site lead to the expectation that dissolution in partially saturated solutions would occur from a zone extending from the surface to a depth of about $50 \mu \mathrm{m}$. In synthetic hydroxyapatite this is manifested as a zone of softening, while in clinical dentistry the zonal dissolution leads to the formation of white spots, which have long been known to be the precursors to cavitation.

The implications of the discovery of this second dissolution site are great. Any studies which are done in an effort to test possible treatment regimens for the clinical reduction of caries must include experiments done under conditions of partial saturation so that the clinically active site is the one being observed. In particular, the effects of fluoride and trace metal ions on enamel dissolution must be studied under conditions of partial saturation to have any clinical meaning.

The discovery of this second site will guide researchers in the right direction in choosing future experiments to better understand the behavior of dental enamel under clinical conditions and hence to develop better regimens for caries prevention and treatment.

\section{SUMMARY}

Previous studies (9) showed that under sink conditions dissolution of human dental enamel and synthetic hydroxyapatite pellets 
could be reasonably well described by a model employing a single dissolution site. This site was not in instantaneous equilibrium with the adjacent solution, but rather had a rate of dissolution characterized by a first-order rate constant. The present investigation reports similar studies carried out under the clinically more relevant conditions of dissolution into partially saturated media, and it is shown that under these conditions dissolution may be described by a site which has properties different from the sink site (9). The properties of this site were consistent with a great number of experiments in which bulk solution conditions were varied. Hence the available evidence indicates that hydroxyapatite crystals have at least two different types of sites that are important in dissolution. A detailed discussion of the two-site model and its implications is contained in a separate paper (2).

\section{REFERENCES}

1. Fox, J. L., Ph.D. Thesis, The University of Michigan, 1977.

2. Fox, J. L., Higuchi, W. I., Fawzi, M. D., and Wu, M. S., J. Colloid Interface Sci. 67, 312 (1978).

3. Higuchi, W. I., Gray, J. A., Hefferren, J. J., and Patel, P. R., J. Dent. Res. 44, 330-341 (1965).

4. Higuchi, W. I., Mir, N. A., Patel, P. R., Becker, J. W., and Hefferren, J. J., J. Dent. Res. 48, 396-409 (1969).

5. Levich, V. G., "Physicochemical Hydrodynamics," chap. 11. Prentice-Hall, New York, 1962.

6. Mir, N. A., and Higuchi, W. I., Arch. Oral. Biol. 14, 901-920 (1969).

7. Moreno, E. C., Gregory, T. M., and Brown, W. E., J. Res. Nat. Bur. Std. 72A, 773-782 (1968).

8. Riddiford, A. C., in "Advances in Electrochemistry and Electrochemical Engineering, Vol. 4, p. 47. (P. Delahay, Ed.), Interscience, New York, 1966.

9. Wu, M. S., Higuchi, W. I., Fox, J. L., and Friedman, M., J. Dent. Res. 55, 496-505 (1976). 